\title{
Studies on the mode of action and the selectivity of insecticides acting on ligand-gated ion channels in the nervous system*
}

\author{
Kazuhiko MATSUDA** \\ Department of Applied Biological Chemistry, School of Agriculture, \\ Kinki University, 3327-204 Naka-machi, 631-8505 Nara, Japan
}

(Accepted February 20, 2009)

\begin{abstract}
The mode of action and the mechanisms for the selectivity of neurotoxic insecticides were investigated using computational chemistry, molecular biology, homology modeling of the target site and X-ray crystallography. Whole-cell, patch-clamp electrophysiology has been used to show that non-competitive antagonists (NCAs), such as 4 '-ethynyl-4- $n$-propylbicycloorthobenzoate (EBOB) and $\gamma$-hexachlorocyclohexane $(\gamma-\mathrm{HCH})$, are more effective blockers of native cockroach (Periplaneta americana) and recombinant house fly (Musca domestica) $\gamma$ aminobutyric acid receptors (GABARs) than glutamate-gated chloride channels (GluCls). Site-directed mutagenesis studies have been used to show that that Ser278 in the second transmembrane region is important for determining the less potent actions of these NCAs on GluCls. The structure-activity relationship and the mechanism of the selectivity of neonicotinoids targeting nicotinic acetylcholine receptors (nAChRs) have also been investigated. Electrophysiological studies show that loops $\mathrm{C}$ and D of insect $\mathrm{nAChRs}$ are critical in determining the selectivity of neonicotinoids, results consistent with the crystal structures of Lymnaea stagnalis acetylcholine binding protein $(L s-\mathrm{AChBP})$ in complex with imidacloprid. Neonicotinoids show diverse actions on nAChRs as measured using voltage-clamp electrophysiology. Single channel recording and X-ray crystallography are helping to elucidate the mechanism of super agonist actions of clothianidin and an analog. (C) Pesticide Science Society of Japan
\end{abstract}

Keywords: Ligand-gated ion channels, Rdl GABA receptors, glutamate-gated chloride channels, neonicotinoids, nicotinic acetylcholine receptors, non-competitive inhibitors.

\section{Introduction}

Cys-loop ligand-gated ion channels (LGICs) mediate fast synaptic neurotransmission in mammals and insects. Cys-loop receptor channels consist of five subunit proteins, each containing a long N-terminal ligand binding domain and four transmembrane (TM) segments. Several classes of insecticides modulate the LGICs, exerting neurotoxicity. It has been established that non-competitive antagonists (NCAs), such as $\gamma$-hexachlorocyclohexane $(\gamma-\mathrm{HCH})$, dieldrin and fipronil, as well as natural product picrotoxinin (PTX) block the chloride channels of ionotropic $\gamma$-aminobutyric acid receptors (GABAR), whereas imidacloporid and related insecticides, referred to as neonicotinoids, selectively bind insect

\footnotetext{
* See Part II for the full Japanese article. Email:kmatsuda@nara.kindai.ac.jp

(C) Pesticide Science Society of Japan
}

nicotinic acetylcholine receptors (insect nAChRs). Despite these achievements, much remains to be discovered concerning the molecular mechanisms underlying the modes of action and selectivity of insecticides. Thus, computational chemistry, molecular biology, homology modeling of the ligand binding domain of the target receptors, and X-ray crystallography have been employed to elucidate the mechanisms.

\section{Structures of GABARs and GluCls and the selectivity of NCAs}

The NCAs ( $\gamma$-HCH, dieldrin, fipronil and PTX) were thought to target only insect GABARs until the $C$. elegans $\mathrm{GluCl}$ genes were cloned and functionally expressed. This finding raised a question as to the selectivity of NCAs to GABARs. Similarly, it was necessary to examine the selectivity of the radioligand 4 '-ethynyl-4- $n$-propylbicycloorthobenzoate (EBOB), which had been used to evaluate the binding affinity of many compounds for GABARs. EBOB and $\gamma$-HCH selec- 
tively blocked GABA-induced responses more effectively than the glutamate-induced responses of $P$. americana neurons. Similar GABAR selectivity was observed for fipronil and PTX when tested on recombinant Musca domestica GABA receptor $\mathrm{Rdl}(\mathrm{MdRdl})$ and $\mathrm{GluCl}(\mathrm{MdGluCl})$ expressed in Xenopus laevis oocytes.

A single amino acid mutation, A302S, is known to reduce the sensitivity of the Drosophila melanogaster Rdl GABA receptor to NCAs. As GluCls possess Ser278 as the corresponding amino acid, this might contribute to the lower NCA sensitivity of GluCls. Thus, the effects of S278A mutation on the blocking actions of NCAs on $\mathrm{MdGluCl}$ have been investigated. For this mutation, the concentration-inhibition curves of $\gamma-\mathrm{HCH}, \mathrm{PTX}$ and fipronil are shifted to left (lower concentrations), suggesting the contribution of Ser278 to the NCA sensitivity of $\mathrm{MdGluCl}$; however, such a shift of the curves can be counteracted by increasing the glutamate concentration. NCAs enhance the response when co-applied with a high concentration of glutamate.

GABARs and GluCls have similar roles in inhibitory neurotransmission and have also been shown to interact; however, the role of such an interaction is unknown. Thus, MdRdl and $\mathrm{MdGluCl}$ were co-expressed in Xenopus laevis oocytes to investigate the changes in their pharmacology. Co-expression of these receptors enhanced the response to GABA of MdRdl with no apparent influence on the concentration-response curve of GABA, suggesting a chaperone-like role of $\mathrm{MdGluCl}$ in the expression of MdRdl.

\section{Mechanism of the selective actions of neonicotinoids}

Neonicotinoids show selectivity to insects over mammals by showing a degree of selectively for insect nAChRs. It is conceivable that this selectivity is attributable to the interactions of neonicotinoid-unique structures with insect-nAChR-unique amino acids or backbones. Computational chemistry indicated that the imidazolidine ring of imidacloprid becomes electron-deficient when the nitro group forms a complex with ammonium. Further calculation using model molecules indicated that the complex was stabilized in an order of methylammonium $>$ pheonol $>$ methanol, suggesting a critical role of nitro group-basic residue interactions in the high binding affinity for insect nAChRs.

Although it is difficult to express insect nAChRs functionally in Xenopus oocytes and cell lines, insect $\alpha$ subunits will form robust, functional nAChRs in Xenopus oocytes and the Drosophila melanogaster cell line S2. Thus, the neonicotinoid sensitivity of Drosophila D $\alpha 2 /$ chicken $\beta 2$ hybrid nAChRs was compared with that of chicken $\alpha 4 \beta 2$ nAChR using twoelectrode voltage-clamp electrophysiology. The hybrid $\mathrm{nAChR}$ is more sensitive to neonicotinoids than $\alpha 4 \beta 2$ nAChR. Similar results have been obtained with Drosophila $\mathrm{D} \alpha 1 /$ chicken $\beta 2$ hybrid $\mathrm{nAChR}$, suggesting the contribution of Drosophila $\alpha$ subunits to selective interactions with neoni- cotinoids.

The ligand binding domain (LBD) is located at the interface of $\alpha$ and non- $\alpha$ subunits but in the case of homomers and $\alpha 9 / \alpha 10$ heteromer, the LBD is generated at the interface of two adjacent $\alpha$ subunits. Somewhat atypically for verbrate nAChRs, the homomer-forming $\alpha 7$ is moderately sensitive to neonicotinoids, so this subunit, as well as $\alpha 4 \beta 2$ and $\mathrm{D} \alpha 2 \beta 2$ nAChRs, was used to identify amino acids influencing neonicotinoid sensitivity to nAChRs.

The LBD consists of 6 loops (loops A, B, C, D, E and F). Of these loops, $\mathrm{A}-\mathrm{C}$ are donated by $\alpha$ subunits, whereas loops D-F are donated by non- $\alpha$ or homomer-forming $\alpha 7$ subunits. G189D and G189E mutations in loop $\mathrm{F}$ of $\alpha 7$ markedly reduced the response to imidacloprid and nitenpyram but G189N and G189Q mutations had scarcely no influence. Such a response-reducing effect of G189D and G189E mutations was not observed for desnitro-imidacloprid (DN-IMI) lacking the nitro group, suggesting that changes in the response to neonicotinoids by the addition of acidic residues to loop $\mathrm{F}$ might result from electrostatic repulsion between the acid residue and the nitro group. The findings also suggested that the introduction of basic residues may enhance the nAChR response to neonicotinoids, but no such basic residues are present in insect non- $\alpha$ subunits; however, I191W mutation, a change of Ile191 to the aromatic residue seen in some insect non- $\alpha$ subunits, in loop $\mathrm{F}$ of $\alpha 7$, enhances the neonicotinoid sensitivity of $\mathrm{nAChR}$.

The water-soluble acetylcholine binding protein AChBP from Lymnaea stagnalis (Ls-AChBP) is homologous to the LBD of $\alpha 7 \mathrm{nAChR}$ and, like the receptor, also forms a homopentamer. Thus, AChBP can be regarded as a surrogate for LBD. The crystal structure of $L s$-AChBP provides an opportunity to examine LBD. The amino acid corresponding to mutated G189 is located in the vicinity of AChBP Gln55 in loop $\mathrm{D}$ corresponding to $\mathrm{G} \ln 79$ of $\alpha 7$. If the acidic residue added to loop $\mathrm{F}$ is located close to the nitro group of neonicotinoids in $\alpha 7 \mathrm{LBD}$, then Gln55 should also be located in the vicinity of the same functional group. Q79E mutation markedly reduced the response of $\alpha 7$ to neonicotinoids, whereas Q79R and Q79K enhanced the response. By contrast, the Q79E mutation slightly enhanced the response to DN-IMI, whereas Q79K and Q79R mutations reduced the response. Since basic residues are preserved at the corresponding site of insect non$\alpha$ subunits, the nitro group-insect selective basic residues in loop D is thought to play a key role in determining the selective actions on insect nAChRs. This has been further examined using $\alpha 4 \beta 2$ and $\mathrm{D} \alpha 2 \beta 2 \mathrm{nAChRs}$ expressed in Xenopus oocytes. The $\beta 2$ amino acids corresponding to $\alpha 7$ Gln 79 is Thr77, so this amino acid has been replaced by the corresponding residue in insect non- $\alpha$ subunits; however, such a single amino acid mutation only slightly affected the concentrationresponse curve of imidacloprid. Thus, both Thr77 and Glu79 have been mutated to the corresponding insect nAChR residues, because Glu79 is likely to interfere electronically 
with the nitro group-basic residue interactions. The T77R; E79V and related double mutations significantly shifted the concentration-response curve of imidacloprid to the left, supporting the notion of the important contribution of the nitro group-loop D basic residue in determining the high affinity for insect $n A C h R s$.

As noted above, insect $\mathrm{nAChR} \alpha$ subunits also possess structural features favorable for interactions with neonicotinoids. Comparing the primary structures of vertebrate and insect $\alpha$ subunits led to the identification of the YXCC motif and long loop B-C interval of insect $\alpha$ subunits as candidates for neonicotinoid-sensitivity determinants. Vertebrate $\alpha 2$ and $\alpha 4$ subunits have a glutamate residue in this motif, whereas insect $\alpha$ subunits have no such acidic residue. Replacing the YECC motif of $\alpha 4$ by the YPCC motif enhanced the $\alpha 4 \beta 2$ response to imidaclorpid, whereas replacing the YPCC motif of $\mathrm{D} \alpha 2$ by the YECC motif reduced the response and shifted the $\mathrm{D} \alpha 2 \beta 2$ concentration-response curve of imidacloprid to the right. Interestingly, the loop $\mathrm{B}-\mathrm{C}$ interval alone had no effects on the concentration-response curve of imidacloprid but when combined with the YXCC motif, it enhanced the shift of the curve induced by replacement of the motif. The $\alpha 4$ $\mathrm{D} \alpha 2$ chimeras were used to further explore the $\mathrm{D} \alpha 2$ subunit features contributing to selective neonicotinoid actions. Measuring the concentration-response curve of chimera nAChRs has identified the region upstream of loop B likely to be involved in such actions.

\section{Diversity of neonicotinoid actions}

Voltage-clamp electrophysiology permits investigation of neonicotinoid actions on nAChRs in terms of affinity and efficacy. Neonicotinoids with an imidazolidine ring are partial agonists of native and recombinant nAChRs. Opening the imidazolidine ring increases the efficacy of compounds. Neonicotinoids containing a nitromethylene unit show higher agonist potency than those containing a nitroimine unit.

Neonicotinoids do not always act as agonists or partial agonists. Imidacloprid reduced the ACh-induced response of nAChRs when tested on cockroach neurons at low concentrations where no agonist responses can be detected. On the other hand, bis-neonicotinoids, consisting of two neonicotinoid units linked with an alkyl chain, showed no agonist action even at $10 \mu \mathrm{M}$ on cockroach neurons, but instead blocked the ACh response in the nanomolar range.

Imidacloprid and clothianidin potentiate the ACh response of $\alpha 4 \beta 2 \mathrm{nAChR}$ when co-applied with $\mathrm{ACh}$, whereas thiacloprid only reduces the response amplitude. The potentiation of the ACh response of $\alpha 4 \beta 2$ is attenuated by increasing ACh concentration, suggesting competitive binding of the neonicotinoids with ACh in this instance. The nitro group of imidacloprid and the cyano group of thiacloprid were exchanged to elucidate the structural basis for potentiation. The "cyano-im- idacloprid" did not potentiate the ACh response, whereas the "nitro-thiacloprid" did, suggesting the contribution of the nitro group to such potentiation.

Among the neonicotinoids, clothianidin and its analog $\mathrm{CH}-$ CTD (a nitromethylene analog of clothianidin) are unique in that they induce a greater maximum response than $\mathrm{ACh}$, not only in hybrid $\mathrm{D} \alpha 2 \beta 2 \mathrm{nAChR}$ expressed in Xenopus oocytes, but also in the native Drosophila larval brain neurons. Single channel recording of the CH-CTD-modified channel revealed that the neonicotinoid opened the channel more frequently than $\mathrm{ACh}$ at the highest conductance state, offering a possible explanation for such a "super agonist" action.

\section{Structural basis of selectivity and diversity of neonicotinoid actions}

To elucidate the structural basis for the selectivity of neonicotinoids as well as the super agonist action of clothianidin, the $L s$-AChBP was co-crystallized with imidacloprid and clothianidin. The crystal structure of $L s$-AChBP in complex with imidacloprid shows that the pyridine nitrogen hydrogen bonds with the backbone of loop E via a water molecule, whereas the imidazolidine ring stacks with Tyr185 in loop C, and the methylene hydrogen of the imidazolidine ring forms $\mathrm{CH}-\pi$ hydrogen bonds with Trp143 in loop B. Furthermore, the nitro group interacts directly with Gln55 in loop D, consistent with the results of site-directed mutagenesis in this loop. One hydrogen atom on the guanidine moiety of clothianidin forms a hydrogen bond with the backbone carbonyl of Trp143, this binding accounting, at least in part, for its super agonist action.

\section{Concluding remarks}

In this study, the selectivity of NCAs to insect GABARs was elucidated and a serine residue of GluCls was found to play a role in determining the lower NCA sensitivity observed when compared to the findings of GABARs. In addition, the $\mathrm{GluCl}$ subunit enhanced the expression of Rdl when co-expressed in Xenopus oocytes.

Site-directed mutagenesis combined with two-electrode voltage-clamp electrophysiology has shown that loops $\mathrm{C}$ and $\mathrm{D}$ are particularly important for determining the high neonicotinoid affinity for insect nAChRs. In addition, electrophysiological experiments have demonstrated diverse neonicotinoid actions. Crystallization of the $L s$-AChBP in complex with imidacloprid and clothianidin offers a structural basis for selective nAChR-neonicotinoid interactions and the super agonist action of clothianidin and its analog.

As pest species-selective insecticides are desired, the overall achievements of this study will help to design such selective compounds from a structural basis. The results are also expected to be useful for predicting mutations that reduce neonicotinoid sensitivity in pests. 